\title{
The Neuroscience of Musical Improvisation
}

By: Roger E. Beaty

Beaty, R.E. (2015). The neuroscience of musical improvisation. Neuroscience \& Biobehavioral Reviews, 51, 108-117.doi: 10.1016/j.neubiorev.2015.01.004

Made available courtesy of Elsevier: http://dx.doi.org/10.1016/j.neubiorev.2015.01.004

***@ $\subseteq$ Elsevier. Reprinted with permission. No further reproduction is authorized without written permission from Elsevier. This version of the document is not the version of record. Figures and/or pictures may be missing from this format of the document. $* * *$

This is the author's version of a work that was accepted for publication in Neuroscience \& Biobehavioral Reviews. Changes resulting from the publishing process, such as peer review, editing, corrections, structural formatting, and other quality control mechanisms may not be reflected in this document. Changes may have been made to this work since it was submitted for publication. A definitive version was subsequently published in Neuroscience \& Biobehavioral Reviews, Volume 51, (2015) DOI: 10.1016/j.neubiorev.2015.01.004

\begin{abstract}
:
Researchers have recently begun to examine the neural basis of musical improvisation, one of the most complex forms of creative behavior. The emerging field of improvisation neuroscience has implications not only for the study of artistic expertise, but also for understanding the neural underpinnings of domain-general processes such as motor control and language production. This review synthesizes functional magnetic resonance imagining (fMRI) studies of musical improvisation, including vocal and instrumental improvisation, with samples of jazz pianists, classical musicians, freestyle rap artists, and non-musicians. A network of prefrontal brain regions commonly linked to improvisatory behavior is highlighted, including the presupplementary motor area, medial prefrontal cortex, inferior frontal gyrus, dorsolateral prefrontal cortex, and dorsal premotor cortex. Activation of premotor and lateral prefrontal regions suggests that a seemingly unconstrained behavior may actually benefit from motor planning and cognitive control. Yet activation of cortical midline regions points to a role of spontaneous cognition characteristic of the default network. Together, such results may reflect cooperation between large-scale brain networks associated with cognitive control and spontaneous thought. The improvisation literature is integrated with Pressing's theoretical model, and discussed within the broader context of research on the brain basis of creative cognition.
\end{abstract}

Keywords: Creativity | Expertise | Music | Improvisation | Premotor | fMRI

Article:

\section{Introduction}


Improvisation is one of the most complex forms of creative behavior. The improvising musician faces the unique challenge of managing several simultaneous processes in real-time-generating and evaluating melodic and rhythmic sequences, coordinating performance with other musicians in an ensemble, and executing elaborate fine-motor movements-all with the overall goal of creating esthetically appealing music. Other forms of artistic performance, while similarly demanding, do not require such spontaneous creativity. The question of how musicians improvise is relevant not only to the psychology of music, it also has implications for the psychology of creativity, as understanding the nature of creativity at a high level of skilled performance may shed light on domain-general processes underlying creative cognition. Improvisation research may also inform basic cognitive neuroscience because it provides a unique look at how acquired expertise shapes brain structure and function.

An increasing number of studies are employing neuroimaging methods to explore the brain basis of spontaneous musical composition, using samples of jazz pianists, classical musicians, freestyle rap artists, and non-musicians. Much of this research has focused on understanding the extent to which brain regions associated with executive control mechanisms underlie improvised behavior. Does improvisation rely on the musician's ability to control the creative process, or rather on his or her ability to "let go" of control and allow spontaneous processes to unfold? This review examines the issue of cognitive control in creative thought with the overarching goal of understanding the cognitive and neural underpinnings of musical improvisation.

\subsection{Pressing's model of improvisation}

Perhaps the most influential model of musical improvisation was developed by Pressing, 1988 and Pressing, 1998. Following in the literature on expert performance (Ericsson et al., 1993), Pressing's theory is grounded in the notion that improvisation is an acquired skill that requires a substantial amount of training to achieve expertise. A large body of research in a range of domains suggests that expertise is achieved through deliberate practice, an individually tailored regimen of intensive training typically undertaken with the guidance of an expert instructor (Ericsson et al., 1993). According to the deliberate practice view, eminence in a domain is rarely achieved without thousands of hours of deliberate practice: Ericsson and colleagues have repeatedly demonstrated that experts typically engage in $10,000 \mathrm{~h}$ of deliberate practice over the course of ten years before achieving eminence in their field (i.e., the "10-year rule"; Simon and Chase, 1973). Recently, however, researchers have emphasized the role of general cognitive abilities (e.g., working memory capacity; Meinz and Hambrick, 2010) and genetic predispositions (Ericsson, 2013 and Tucker and Collins, 2012) in explaining expert performance, thus providing support for the notion that practice is "necessary but not sufficient" for high-level performance (Hambrick et al., 2014 and Hambrick and Meinz, 2011).

Domain-specific expertise seems especially relevant to musical improvisation. In addition to the physical and psychological constraints common to other domains of skilled performance, jazz musicians must perform under extraordinary temporal constraints. Improvising requires the 
simultaneous execution of several processes in real-time, including sensory and perceptual encoding, motor control, performance monitoring, and memory retrieval, among others (Pressing, 1988). Deliberate practice automates some of these processes, freeing attentional resources for other higher-order processes (e.g., generating and evaluating musical ideas). In the absence of such improvisational fluency, the improviser will have difficulty effectively interacting with other members of an ensemble and exerting control over the development of his or her performance.

According to Pressing's model, improvisational expertise involves the interplay between referent processes and a domain-specific knowledge base. Referents consist of cognitive, perceptual, or emotional processes; the knowledge base consists of hierarchical knowledge structures stored in long-term memory (Pressing, 1988). Pressing described referents as a series of well-rehearsed retrieval cues that are deployed during performance, minimizing processing demands and guiding idea generation. Referents interact with procedural and declarative information stored in a domain-specific knowledge base. Through deliberate practice, musicians build a database of generalized motor programs, which can be fluently accessed and executed during performance.

Another component of Pressing's model is perceptual feedback and error correction. These processes allow the improviser to minimize the distance between intended and actual performance (Pressing, 1988 and Pressing, 1998). Pressing distinguishes between short-term (ongoing motor movements) and long-term (decision making and response selection) feedbackboth of which are essential for improvisational fluency. In contrast to "open-loop" theories of skilled performance, which consist of a simple input, processing, and output procedure, Pressing advances a "closed loop" model, which extends open-loop models by including feedback integration within the system. Ongoing performance is thus monitored by comparing actual output with intended output, and future performance is adjusted accordingly.

Pressing (1988) conceptualized improvisation as a series of generative and evaluative processes. Although these processes involve some level of cognitive control and conscious monitoring, Pressing emphasized the role of automatized motor processes and routines (e.g., well-rehearsed action sequences). Because of the high demands on information processing and decision-making, Pressing argued that improvisational fluency relies on automatized processes that require minimal conscious attention. The extent to which creative thought relies upon such top-down and bottom-up processes remains a point of debate in the literature on musical improvisation as well as in the literature on domain-general creative cognition (cf. Abraham, 2014, Beaty et al., 2014c, Jung et al., 2013, McMillan et al., 2013,Mok, 2014 and Sowden et al., 2014).

\subsection{Domain-general creative cognition}

The study of musical improvisation provides an opportunity to investigate creativity at a high level of skilled performance. Although improvisation research has traditionally been restricted to the field of musicology, it is also of growing interest to researchers in the field of creativity 
science. Several literature reviews and meta-analyses on the neuroscience of creativity include studies on musical improvisation (e.g., Dietrich and Kanso, 2010; Gonen-Yaacovi et al., 2013). Moreover, results from behavioral and neurophysiological research suggest that improvisation taps domain-general processes such as divergent thinking (Beaty et al., 2013) and cognitive flexibility (de Manzano and Ullén, 2012b).

The cognitive and neural basis of creative thought has been a topic of increasing empirical interest. Much of this work has employed divergent thinking tasks, the most common of which is the alternate uses task. Such tasks require the generation of novel uses for everyday objects (e.g., a brick), and they are typically scored in terms of fluency (the number of ideas) and originality (the creative quality of ideas). A growing body of evidence suggests that individual differences in divergent thinking reflect a domain-general creative ability: performance on divergent thinking tasks has been shown to predict both past and future creative achievements (Jauk et al., 2014, Plucker, 1999 and Torrance, 1988). Moreover, a recent study found that divergent thinking ability in jazz students predicted expert ratings of improvisational performance, controlling for cumulative lifetime practice hours (Beaty et al., 2013).

Researchers have long conceptualized creative cognition as an associative process that passively unfolds in long-term memory (Mednick, 1962). In general, such work suggests that creative ideas largely result from the spreading activation of concepts in semantic networks, and that individual differences in creative ability result from variation in the structural organization of semantic memory. More recently, researchers have explored the cognitive processes involved in divergent thinking, the ability to generate several novel solutions to open-ended problems. Behavioral (Barr et al., 2014, Beaty and Silvia, 2012, Beaty and Silvia, 2013 and Benedek et al., 2014c; Lee and Therriault, 2013; Nusbaum and Silvia, 2011 and Nusbaum et al., 2014; Silvia et al., 2013) and neurophysiological (Beaty et al., 2014a, Beaty et al., 2014b, Benedek et al., 2014, Fink and Benedek, 2014, Fink et al., 2009 and Gonen-Yaacovi et al., 2013) research suggests that higher-order cognitive processes underlie creative cognition, such as controlled memory retrieval, pre-potent response inhibition, fluid intelligence, and working memory capacity. Such processes are hypothesized to support creative thought by providing the executive control needed to manage complex search processes and inhibit salient conceptual information that can interfere with idea generation (Beaty and Silvia, 2012).

Recent behavioral and neuroimaging research suggests that musical improvisation may also recruit domain-general processes (Beaty et al., 2013 and de Manzano and Ullén, 2012b). Although improvisational expertise, like any other acquired skill, is undoubtedly a function of domain-specific training, there is evidence to suggest that divergent thinking and domain-general mental flexibility play an important role. A growing literature provides support for the notion that creative cognition recruits brain regions linked with executive processes (e.g., strategic memory retrieval and pre-potent response inhibition). Nevertheless, the role of executive processes in creative thought remains a point of contention in both the general creativity literature and the literature on musical improvisation. 


\subsection{Organization of the review}

The following review synthesizes neuroimaging research on musical improvisation. Table 1 summarizes the studies included in the review; Fig. 1 and Fig. 2 depict activation foci resulting from positive task contrasts in select fMRI studies of improvisation. Pressing's (1988) theoretical model of improvisation is used as a guiding framework for interpreting the brain imaging literature. The literature is also framed within the ongoing debate on whether creativity arises from controlled cognitive processes. Special attention is given to neuroimaging research on domain-general creativity, in light of recent evidence pointing to an overlap between general creativity and musical improvisation. The review has three broad goals: (1) to synthesize the improvisation neuroscience literature, (2) to provide a conceptual framework for interpreting brain imaging results on improvisation, and (3) to integrate these findings with the literature on domain-general creative thought.

Table 1. Summary of methods used to study improvisation with fMRI.

\begin{tabular}{|l|l|l|l|l|l|}
\hline Study & Year & $\begin{array}{l}\text { \# of } \\
\text { subjects }\end{array}$ & Type of musician & Contrast & Response type \\
\hline $\begin{array}{l}\text { Bengtsson } \\
\text { et al. }\end{array}$ & 2007 & 11 & $\begin{array}{l}\text { Classically-trained } \\
\text { pianists (graduate) }\end{array}$ & $\begin{array}{l}\text { Improvise > Memory } \\
\text { retrieval }\end{array}$ & $\begin{array}{l}\text { MRI- } \\
\text { compatible } \\
\text { keyboard (12 } \\
\text { keys) }\end{array}$ \\
\hline $\begin{array}{l}\text { Berkowitz } \\
\text { and Ansari }\end{array}$ & 2008 & 13 & $\begin{array}{l}\text { Classically-trained } \\
\text { pianists } \\
\text { (undergraduate) }\end{array}$ & $\begin{array}{l}\text { Improvise } \\
\text { melody > Improvise } \\
\text { rhythm }\end{array}$ & $\begin{array}{l}\text { MRI response } \\
\text { box (5 buttons) }\end{array}$ \\
\hline $\begin{array}{l}\text { Berkowitz } \\
\text { and Ansari }\end{array}$ & 2010 & 26 & $\begin{array}{l}\text { Classically-trained } \\
\text { pianists \& non- } \\
\text { musicians } \\
\text { (undergraduate) }\end{array}$ & $\begin{array}{l}\text { Between-subjects } \\
\text { design }\end{array}$ & $\begin{array}{l}\text { Conventional } \\
\text { MRI response } \\
\text { box (5 buttons) }\end{array}$ \\
\hline $\begin{array}{l}\text { de } \\
\text { Manzano } \\
\text { and Ullén } \\
\text { and Ullén }\end{array}$ & $2012 \mathrm{a}$ & 17 & $\begin{array}{l}\text { Classically-trained } \\
\text { pianists } \\
\text { (undergraduate) }\end{array}$ & $\begin{array}{l}\text { Improvise } \\
\text { melody > Improvise } \\
\text { rhythm }\end{array}$ & $\begin{array}{l}\text { MRI- } \\
\text { compatible } \\
\text { keyboard (12 } \\
\text { keys) }\end{array}$ \\
\hline $\begin{array}{l}\text { de } \\
\text { pianists } \\
\text { (undergraduate) }\end{array}$ & 18 & $\begin{array}{l}\text { Classically-trained } \\
\text { Improvise > Random }\end{array}$ & $\begin{array}{l}\text { MRI- } \\
\text { compatible } \\
\text { keyboard (12 } \\
\text { keys) }\end{array}$ \\
\hline
\end{tabular}




\begin{tabular}{|l|l|l|l|l|l|}
\hline $\begin{array}{l}\text { Donnay et } \\
\text { al. }\end{array}$ & 2014 & 11 & $\begin{array}{l}\text { Jazz musicians } \\
\text { (professional) }\end{array}$ & $\begin{array}{l}\text { Improvise }>\text { Memory } \\
\text { retrieval }\end{array}$ & $\begin{array}{l}\text { MRI- } \\
\text { compatible } \\
\text { keyboard (35 } \\
\text { keys) }\end{array}$ \\
\hline $\begin{array}{l}\text { Limb and } \\
\text { Braun }\end{array}$ & 2008 & 6 & $\begin{array}{l}\text { Jazz musicians } \\
\text { (professional) }\end{array}$ & $\begin{array}{l}\text { Improvise }>\text { Memory } \\
\text { retrieval }\end{array}$ & $\begin{array}{l}\text { MRI- } \\
\text { compatible } \\
\text { keyboard (35 } \\
\text { keys) }\end{array}$ \\
\hline Liu et al. & 2012 & 12 & $\begin{array}{l}\text { Freestyle rap } \\
\text { musicians } \\
\text { (professional) }\end{array}$ & $\begin{array}{l}\text { Improvise }>\text { Memory } \\
\text { retrieval }\end{array}$ & $\begin{array}{l}\text { Optical } \\
\text { microphone }\end{array}$ \\
\hline Pinho et al. & 2014 & 39 & $\begin{array}{l}\text { Pianists with } \\
\text { various levels of } \\
\text { improvisational } \\
\text { expertise }\end{array}$ & $\begin{array}{l}\text { Improvise }>\text { Rest } \\
\text { MRI- } \\
\text { compatible } \\
\text { keyboard (12 } \\
\text { keys) }\end{array}$ \\
\hline
\end{tabular}

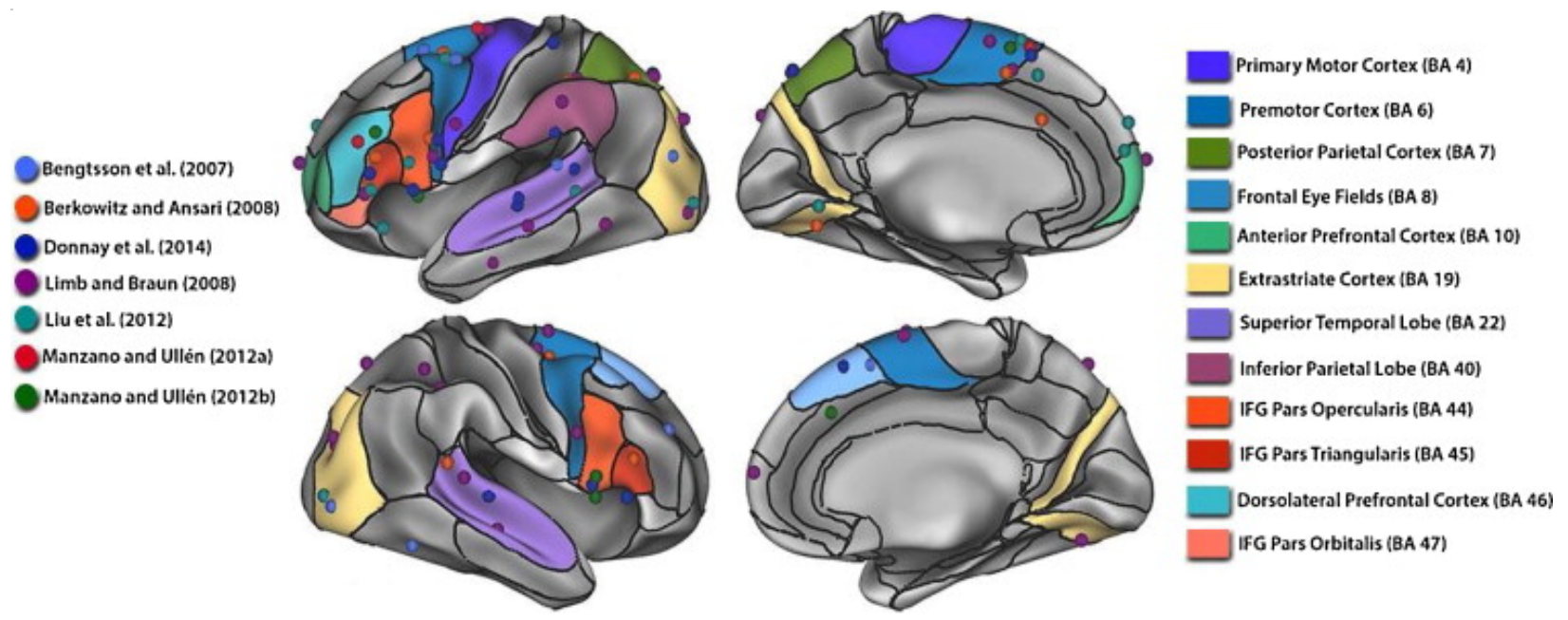

Fig. 1. Visualization of activation foci reported in select studies of improvisation. Note: Activation foci and Brodmann area borders were overlaid on an inflated brain using the Connectome Workbench. Highlighted regions indicate areas with activation reported in at least two of the seven studies included in the figure. For clarity, only positive activation foci are displayed. Studies that report only deactivations ( Berkowitz and Ansari, 2010) and only functional connectivity results ( Pinho et al., 2014) were not included. 


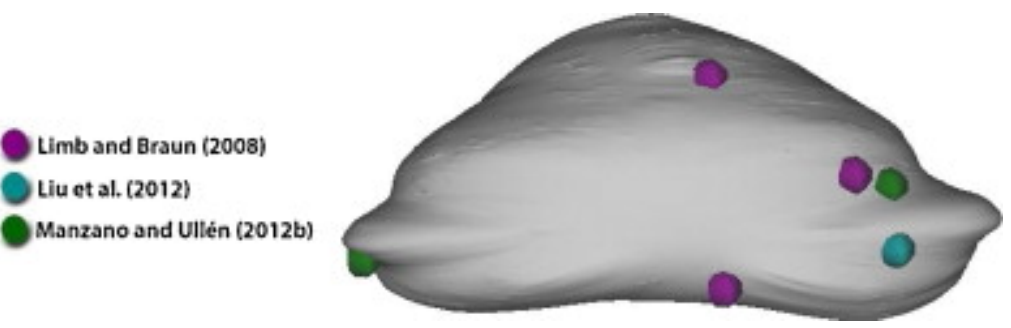

Fig. 2. Visualization of cerebellar activation foci reported in select studies of improvisation. Note: Activation foci are overlaid on an inflated cerebellum using the Connectome Workbench. A posterior view of the cerebellum is depicted.

A variety of experimental approaches and participant samples have been employed to study improvisation (see Table 1). Several notable differences in task design are apparent in the literature: while some studies contrasted improvisation with memory retrieval (Bengtsson et al., 2007 and Limb and Braun, 2008), others contrasted specific modes of improvisation, such as melodic and rhythmic (Berkowitz and Ansari, 2008,Berkowitz and Ansari, 2010 and de Manzano and Ullén, 2012a). Moreover, some researchers have been interested in analyzing the effects of musical expertise (e.g., Berkowitz and Ansari, 2008 and Pinho et al., 2014) and collaboration (Donnay et al., 2014) on brain activity during improvisation. Such methodological differences are important to consider when comparing results across studies.

The review is organized by the main experimental paradigms used to study improvisation. I begin by summarizing the methods and results of studies within each paradigm (e.g., improvisation vs. memory retrieval), noting similarities and differences within and between task paradigms. I then draw some parallels between the improvisation literature and neuroimaging research on domain-general cognitive and creative abilities. In the Discussion, I highlight some emerging trends and conflicting results in improvisation research, focusing on methodological variability as a possible explanation for discrepant findings. Finally, I conclude with some potential directions for future research.

\section{Experimental methods and results}

\subsection{Improvisation and memory retrieval}

A common approach in the improvisation literature is to contrast improvisation with memory retrieval. Such designs allow researchers to identify brain regions involved in the spontaneous composition of novel melodic sequences, while controlling for the influence of simply recalling previously performed sequences from memory. Limb and Braun (2008) conducted one of the earliest studies that used this approach. Professional jazz musicians were asked to memorize a novel melody before the study. The experimental paradigm involved performing musical sequences on an MRI-compatible keyboard while a pre-recorded jazz rhythm section played through headphones in the scanner. Participants were cued to perform the memorized melody, freely improvise over the pre-recorded rhythm, play a one-octave scale, or improvise. Compared 
to memory retrieval, improvisation was related to activation of a distributed network of brain regions, including the left inferior frontal gyrus (IFG), anterior cingulate cortex (ACC), and the medial prefrontal cortex (MPFC), among several others. Interestingly, the authors reported widespread deactivation of regions within the frontal lobes, including the lateral orbitofrontal cortex (LOFC) and the dorsolateral prefrontal cortex (DLPFC). DLPFC deactivation was interpreted as reflecting a suspension of inhibitory or conscious monitoring processes, while MPFC activity was thought to reflect self-generated, stimulus independent cognitive processes.

A similar experimental approach was employed by Bengtsson et al. (2007). However, this study differed from Limb and Braun (2008) in terms of the memory retrieval condition employed: instead of requiring participants to recall a single, overlearned melodic sequence, Bengtsson et al. asked participants to retrieve self-generated melodies that were previously improvised in the scanner. In addition, improvisation was constrained to modifying simple, eight-bar melodic sequences presented, whereas other studies permitted largely unconstrained improvisation (e.g., Donnay et al., 2014, Limb and Braun, 2008 and Liu et al., 2012). Bengtsson and colleagues reported improvisation-related activity in several prefrontal regions, including the DLPFC, right pre-SMA, and bilateral dorsal premotor cortex (PMD). The authors suggest that such activity reflects increased demands on selective retrieval and inhibitory mechanisms, as improvisation involves selecting responses among a set of competing alternatives; prefrontal regions may also be involved in maintaining higher-order generative strategies (cf. Pressing, 1998). Thus, in contrast to Limb and Braun (2008), Bengtsson et al. suggest that improvisation places greater demands on cognitive and motor control systems.

Researchers have also been interested in exploring the neural correlates of vocal improvisation (e.g., Brown et al., 2006 and Liu et al., 2012). Liu and colleagues recruited professional freestyle rap artists to vocally improvise during functional imaging. The authors used a memory retrieval task as a baseline to contrast with improvisation. Similar to the experimental designs of Limb and Braun (2008), participants recited the lyrics from a prerecorded audio sample composed by the authors, or they spontaneously improvised lyrics, using the same instrumental recording. Results showed improvisation-related activation of the pre-SMA, the dorsal premotor cortex (PMD), and the left IFG, among several other regions. Notably, the authors also reported decreased activation of the DLPFC and increased activation of the MPFC. This pattern mirrors the results of Limb and Braun’s (2008) study of instrumental improvisation that suggested less involvement of executive control mechanisms, although several prefrontal regions associated with executive control showed increased activity during improvisation (i.e., pre-SMA, PMD, and IFG).

Such seemingly discrepant findings have led to different interpretations on the role of cognitive control in improvisation. Limb and Braun (2008) interpreted DLPFC deactivation as reflecting a suspension of inhibitory or conscious monitoring processes. Moreover, activation of the MPFC was thought to reflect self-generated, stimulus independent cognitive processes-hallmark 
features of the default mode network (Andrews-Hanna et al., 2014). This network shows a correlated pattern of functional connectivity in the absence of external task demands, thus researchers hypothesize that its activation reflects internally-directed attention (Buckner and Carroll, 2007). Default mode activity has also been associated with mind-wandering (AndrewsHanna, 2012 and O'Callaghan et al., 2015), theory of mind reasoning (Buckner and Carroll, 2007), and mental simulation (e.g., episodic future thinking; Schacter et al., 2012). According toLimb and Braun (2008), activation of default mode regions during improvisation indicates internally-driven, self-referential mechanisms, which may allow the improviser to suspend conscious monitoring and enter a "flow-like" state.

This pattern of results was largely similar to Liu and colleagues' study of lyrical improvisation. Both studies reported increased activity of the MPFC and decreased activity of the DLPFC. Such findings supported the authors' contention that spontaneous composition involves the suppression of executive control systems and activation of default mode regions. But the results of both studies also point to a degree of cognitive control during improvisation, as both Liu et al. (2012) and Limb and Braun (2008) reported activation of the ACC, SMA, PMD, and IFGregions associated with cognitive and motor control.

\subsection{Melodic and rhythmic improvisation}

Pressing (1988) conceptualized improvisation as a dynamic interplay between several processes, including the generation, evaluation, and execution of novel motor sequences. Investigating such a multi-component processes poses challenges for researchers attempting to isolate its neural correlates. The experiments described above contrasted relatively unconstrained improvisation with some form of basic memory retrieval (e.g., Limb and Braun, 2008 and Liu et al., 2012). Although these approaches tend to preserve the complexity and ecological validity of improvisation compared to others, they also typically yield a large number of significant activation peaks, raising questions about the role of specific sub-processes (Berkowitz, 2010). A more nuanced approach has been to examine different modes of improvisation, such as melodic and rhythmic improvisation.

Berkowitz and Ansari (2008) employed experimental conditions that varied in terms of melodic and rhythmic constraints. The authors used tasks that allowed for more or less spontaneous behavior within each mode of improvisation. In a fixed rhythm and free melody condition, for example, rhythm was constrained by a metronome and the notes were freely chosen by the participants; this permitted an analysis of brain activity related to melodic improvisation, controlling for the influence of rhythmic variability. Results showed that improvising novel melodic patterns was related to activation of bilateral dorsal PMD, the ACC, and the right supramarginal gyrus (SMG); melodic improvisation was also related to the deactivation of several brain regions, including the right angular gyrus, the right superior frontal gyrus, and bilateral posterior cingulate. In contrast, rhythmic improvisation was related to increased activity in the ACC, the left IFG, bilateral PMD, and the left superior and inferior parietal lobules (IPL). 
A conjunction analysis, which analyzed the common activity of melodic and rhythmic improvisation, found significant activation in the left inferior frontal gyrus (IFG), PMD, and rostral ACC. Berkowitz and Ansari (2008) thus revealed common and distinct neural correlates of melodic and rhythmic improvisation, providing greater insight into general and specific aspects of spontaneous composition.

Several prefrontal and parietal brain regions have often been associated with improvised performance. Two of the most frequently implicated regions are the pre-SMA and the PMD, regions involved in the coordinated selection of novel motor sequences (Beudel and de Jong, 2009). These regions were among the most commonly activated in studies contrasting improvisation with memory retrieval (e.g., Bengtsson et al., 2007 and Liu et al., 2012). Although these regions appear to be responsible for similar processes, past research suggests subtle differences in their functional roles in motor behavior, which has important implications for understanding their contribution to musical improvisation. On the one hand, the pre-SMA is commonly implicated in the generation, perception, and learning of temporal-motor sequences (Bengtsson et al., 2004, Bengtsson et al., 2009, Karabanov et al., 2009 and Schubotz and von Cramon, 2001). On the other hand, the PMD is commonly implicated in tasks involving the generation, perception, and learning of spatial-motor sequences ( Bischoff-Grethe et al., 2004 and Bengtsson et al., 2004).

In light of the differential functions of the pre-SMA and the PMD, de Manzano and Ullén (2012a) sought to determine their unique contributions to musical improvisation by manipulating spatial and temporal aspects of spontaneous composition. Similar to Berkowitz and Ansari (2008), this experiment involved constraining either melodic or rhythmic improvisation. Results showed that melodic improvisation was associated with increased activity in both the left PMD and the left pre-SMA; rhythmic improvisation was also associated with increased activity in the left pre-SMA. The role of the pre-SMA in improvisation was further supported by de Manzano and Ullén (2012b), who explored whether musical improvisation taps similar neural substrates as pseudo-random response generation (PRG)-a domain-general measure of cognitive flexibility that requires top-down control to generate novel motor sequences (Beudel and de Jong, 2009).de Manzano and Ullén (2012b) asked participants to spontaneously compose melodies or press the keys of an MRI-compatible keyboard in a random fashion. A conjunction analysis revealed increased activation in bilateral IFG, bilateral pre-SMA, left DLPFC, right ACC, bilateral insula, and bilateral cerebellar regions. Interestingly, an analysis exploring the brain activity unique to improvising notes compared to pseudo-random generation did not reveal any significant differences, suggesting widespread overlap between the two conditions. The reverse contrast, however, revealed several areas related to random generation, including the right temporoparietal junction (TPJ), medial and lateral premotor areas, the DLPFC, and the cerebellum.

The results of de Manzano and Ullén (2012a) and Berkowitz and Ansari (2008) suggest that the premotor cortex plays a central role in melodic and rhythmic improvisation. Across both studies, 
the dorsal PMD showed robust activity during conditions requiring spontaneous generation of rhythmic sequences. de Manzano and Ullén (2012b) conclude that melodic improvisation and pseudo-random motor movements recruit some of the same neural mechanisms. Similar to other studies of melodic and rhythmic improvisation, the pre-SMA was found to play a critical role. This notion was further reinforced by activation of other regions involved in executive processes, such as the left DLPFC, right ACC, and bilateral IFG. Activation of prefrontal regions were also reported in studies contrasting improvisation and memory retrieval (e.g., Bengtsson et al., 2007 and Donnay et al., 2014).

Berkowitz and Ansari (2008) provide support for several of Pressing, 1988 and Pressing, 1998 theoretical predictions. Pressing conceptualized improvisation as the generation, selection, and execution of novel melodic and motor sequences, processes that appear to correspond to the functional roles of brain regions reported in Berkowitz and Ansari (2008). Specifically, the left IFG is associated with performance on tasks that require controlled retrieval from long-term memory (e.g., verbal fluency; Badre et al., 2005 and Hirshorn and Thompson-Schill, 2006). Moreover, Berkowitz and Ansari (2008) reported activation of the ACC during both melodic and rhythmic improvisation. The ACC is associated with conflict monitoring (Botvinick et al., 2004), voluntary selection (Forstmann et al., 2006), and decision making (Walton et al., 2004)-all of which are components of Pressing's (1988) model of improvisation.

\subsection{The role of musical expertise}

To what extent does improvisational expertise influence the neural correlates of spontaneous performance? Pressing (1988) argued that expertise was critical to improvisational fluency. Because improvisation places substantial demands on information processing, Pressing viewed expertise as a means to automate low-level sensorimotor and cognitive processes so that attention could be allocated to higher-order performance goals. Berkowitz and Ansari (2010) provided seminal evidence that musical expertise affects brain mechanisms involved during improvisation. The authors used the same experimental paradigm as Berkowitz and Ansari (2008) to analyze the effects of musical expertise in improvisation. However, the key difference was the sample, which now included a second control group of undergraduate nonmusicians. Analysis of the main effect of melodic improvisation revealed that the only significant difference between musicians and non-musicians was a deactivation of the right temporoparietal junction (TPJ) in musicians. The ability of musicians to deactivate the right TPJ during improvisation may thus reflect neurophysiological changes associated with acquired expertise.

Other researchers have sought to determine the role of improvisational expertise by studying pianists with a variety of improvisational experience (Pinho et al., 2014). Pinho and colleagues contrasted brain activation during improvisation with activation at rest, and correlated this activity with self-reported lifetime improvisation hours. Interestingly, improvisational expertise was negatively correlated with activation in a right-lateralized network of brain regions, 
including the DLPFC, IFG, anterior insula, and angular gyrus. The authors also specified six seed regions of interests (ROIs) based on activations reported in past work (de Manzano and Ullén, 2012a and de Manzano and Ullén, 2012b): bilateral DLPFC, bilateral dorsal premotor area, and bilateral pre-SMA. A functional connectivity analysis revealed extensive expertiserelated positive connectivity between the prefrontal seed ROIs and other regions of the brain. Most notably, experts showed greater functional connectivity between the DFLPC and premotor cortex during improvisation (i.e., bilateral PMD and pre-SMA). Premotor seed regions also showed widespread connectivity across the cortex and within regions of the cerebellum.

Pinho and colleagues interpret their findings as evidence in support for Pressing's (1988) model of improvisation. They argue that the relative deactivation of executive control networks in experts may correspond to an overall automation of domain-specific cognitive processes, and increased functional connectivity between frontal regions may translate to more efficient access to pre-learned motor patterns and generative strategies stored in long-term memory. Overall, these findings are consistent with Pressing's (1988) position regarding the function of acquired expertise in improvisation-as expertise increases, processing demands should be minimized, allowing attention to be allocated to higher-order goals and performance monitoring.

Berkowitz and Ansari (2010) found that musical expertise was related to a deactivation of the right TPJ. The right TPJ lies in close proximity to the right angular gyrus, a region also found to be deactivated during improvisation in Berkowitz and Ansari (2008). The right TPJ is part of the ventral attention network (Corbetta and Shulman, 2002), which is comprised of the right TPJ and ventral frontal cortex. Suppression of this network is thought to reflect top-down control during tasks requiring focused internal attention (Corbetta et al., 2008 and Corbetta and Shulman, 2002). Modulation of the ventral attention network may provide "task shielding" by filtering or inhibiting information unrelated to the task at hand (Dreisbach and Haider, 2009); deactivation of nodes within the ventral attention network may therefore correspond to internally-directed attention during creative idea production (cf. Benedek et al., 2014). Deactivation of the right TPJ has also been reported in studies of divergent thinking (e.g., Benedek et al., 2014b and Fink et al., 2009), creative writing (Howard-Jones et al., 2005), and product design (Kowatari et al., 2009). Thus, in the context of Berkowitz and Ansari (2010), deactivation of the right TPJ may reflect a greater ability of musicians to focus attention on internal processes during improvisation (e.g., generation and evaluation of novel musical sequences; Pressing, 1988).

\subsection{Collaborative improvisation}

Improvisation often occurs in group settings with multiple artists contributing to a common esthetic work. Collaborative improvisation is notably different from solo improvisation in terms of performance monitoring and perceptual feedback. An individual's performance must be integrated with multiple streams of sensory information (Pressing, 1988). The improviser must therefore allocate considerable attentional resources to both internal (e.g., generative) and external (e.g., communicative) sources. Recently, Donnay and colleagues explored the neural 
correlates of collaborative improvisation in a sample of professional jazz pianists (Donnay et al., 2014). The experimental procedure involved "trading fours" with an experimenter (also a jazz musician) in a nearby control room; participants and the experimenter took turns performing four-bar musical segments-a common mode of improvisation in jazz ensembles. Participants used an MRI-compatible piano keyboard and were given headphones to listen to their own and the experimenters' musical performance. A pre-recorded rhythm section also streamed through the headphones. Participants alternated between the experimental conditions of interest: playing a scale, performing a memorized melody sequence, or improvising; rhythm was constrained to one note per beat.

Compared to performing the memorized melody, improvising was related to increased activation of the pre-SMA, bilateral IFG, bilateral DLPFC, and the posterior superior temporal gyrus (STG); improvising was also related to deactivation of bilateral angular gyrus. The authors also conducted a functional connectivity analysis to determine whether bilateral IFG showed greater functional connectivity with other regions during improvisation. This analysis revealed significantly greater positive connectivity between the left and right IFG. Bilateral IFG activity was also negatively correlated with activity in the STG, and the left IFG was anticorrelated with bilateral angular gyrus during the improvisation condition.

The results of Donnay et al. (2014) provide an interesting contrast to the earlier work of Limb and Braun (2008). In Limb and Braun (2008), musicians showed deactivation of the DLPFC, suggesting less attention control during improvisation. Moreover, regions of the default mode network were found to be more relevant to improvisation in Limb and Braun (2008). Donnay et al. (2014), in contrast, reported increased activation of the DLPFC during collaborative improvisation. The authors note, however, that the nature of collaborative improvisation appears to be different from solo improvisation, which was of interest in Limb and Braun (2008). They suggest that collaborative improvisation involves a distinct type of musical communication that requires greater demands on performance monitoring.

\section{Discussion}

\subsection{Improvisation and domain-general processes}

Neuroimaging studies have shed light on the role of domain-specific training on brain activation during improvisation (e.g., Berkowitz and Ansari, 2010 and Pinho et al., 2014). But to what extent does improvisation involve domain-general processes? de Manzano and Ullén (2012b) found that generating pseudo-random motor sequences activated the same brain regions as improvising new melodic sequences. This suggests that generating musical ideas may share similar underlying processes as those involved in general cognitive flexibility. The literature on general creativity provides further evidence for a relationship between improvisation and domain-general creative cognition. Neuroimaging studies of divergent thinking, for example, consistently report activation of the left IFG during tasks involving idea generation (for a review, 
see Gonen-Yaacovi et al., 2013). Several studies implicate this region in processes involving the generation and evaluation of candidate ideas retrieved from long-term memory (Badre et al., 2005). Improvisation and divergent thinking may thus recruit selective retrieval mechanisms common to the left IFG, providing further support for the theoretical predictions of Pressing, 1988 and Pressing, 1998.

The left IFG has been implicated in nearly all of the studies described above (e.g., Berkowitz and Ansari, 2008, Donnay et al., 2014 and de Manzano and Ullén, 2012b). In de Manzano and Ullén (2012b), for example, a conjunction analysis found that both improvisation and pseudo-random key pressing activated the left IFG, as well as the left pre-SMA, the left DLPFC, bilateral insular cortex, and bilateral cerebellum. A similar pattern was also reported by Berkowitz and Ansari (2008), who found overlapping activation in the left IFG during conditions requiring rhythmic and melodic improvisation. A large body of research points to an important role of the left IFG in controlled memory retrieval (Corbetta et al., 2008 and Corbetta and Shulman, 2002). Much of this work has used verbal fluency tasks to assess the neural correlates of retrieval from long-term memory. Verbal fluency tasks are often used to assess deficits in executive functioning (Troyer et al., 1998). Such tasks are thought to require attention control and strategic access to memory (Unsworth and Engle, 2007). The left IFG seems to be especially involved when demands on retrieval increase, such as when competing representations in semantic memory are high and when switching between semantic categories is required (Hirshorn and Thompson-Schill, 2006). Researchers hypothesize that activation of the left IFG during improvisation reflects similar retrieval mechanisms (e.g.,Berkowitz, 2010). Generating melodic sequences, for example, requires the dynamic interaction between higher-order generative processes and a domainspecific knowledge (Pressing, 1998). In this context, the left IFG may support the generation, evaluation, and selection of to-be-performed musical sequences.

The left IFG is often implicated in fMRI studies of divergent thinking. Divergent thinking is a domain-general creative thought process that involves generating ideas in response to openended prompts (e.g., inventing alternate uses for common objects). A recent review of $34 \mathrm{fMRI}$ studies of divergent thinking found that the left IFG was among the most strongly activated during tasks involving creative idea generation (Gonen-Yaacovi et al., 2013). In addition, a recent resting-state fMRI study showed greater functional connectivity between the left IFG and default mode regions in individuals of high divergent thinking ability (Beaty et al., 2014a and Beaty et al., 2014b). The left IFG therefore appears to be important for a range of cognitive processes requiring controlled search from long-term memory, including musical improvisation and divergent thinking.

Further evidence for a role of the inferior prefrontal cortex comes from a recent study on creative drawing (Ellamil et al., 2012). This work examined the neural mechanisms underlying generative and evaluative processes related to drawing ability in a sample of undergraduate art students. Overall, compared to evaluation, generation was associated with increased activation of the left IFG, bilateral premotor cortex, inferior and superior parietal lobes, and bilateral medial temporal 
lobes; compared to generation, evaluation was associated with greater activity in several regions within executive control and default mode networks. A functional connectivity analysis further revealed greater functional coupling between executive and default networks during evaluation, pointing to a greater cooperation between controlled and spontaneous thought processes (cf. Beaty et al., 2014a, Beaty et al., 2014b, McMillan et al., 2013 and Mok, 2014).

A recent behavioral study suggests that musical improvisation may tap individual differences in domain-general creative cognition (Beaty et al., 2013). In this study, semi-professional jazz musicians were asked to improvise with a trio; they also completed a questionnaire regarding their instrumental practice regimen, as well as an alternate-uses divergent thinking task to assess general creative thinking ability. The improvisations were recorded and scored for creative quality by a team of expert raters. As expected, the number of practice hours was the strongest predictor of improvisation scores. But divergent thinking also predicted improvisation scores, even after controlling for practice hours. The results of this study and other recent work (e.g., Benedek et al., 2014a and Lewis and Lovatt, 2013) point to a potential overlap between the ability to generate creative ideas in general and the ability of jazz musicians to generate novel musical sequences.

Pressing (1988) conceptualized improvisation as the interaction between higher-order referent processes and long-term memory. The neuroimaging literature on improvisation consistently implicates brain regions associated with cognitive control (e.g., the left IFG and the DLPFC); the literature also points to an overlap between domain-specific musical improvisation and domaingeneral cognitive processes (e.g., de Manzano and Ullén, 2012b). Taken together, a growing body of research supports Pressing, 1988 and Pressing, 1998 model of improvisation and further suggests that improvisation may rely on general creative processes.

\subsection{The cognitive control of creative behavior}

One point of contention in the literature is whether improvisation is under the deliberate control of the improviser. On the one hand, several studies implicate brain regions associated with working memory capacity (DLPFC), controlled memory retrieval (left IFG), and volitional motor control (PMD; Bengtsson et al., 2007, de Manzano and Ullén, 2012a and Donnay et al., 2014). On the other hand, there is evidence to suggest that such processes are less involved during improvisation (Limb and Braun, 2008), and that improvisational expertise corresponds to decreased activity within executive control regions (Pinho et al., 2014). Limb and Braun (2008), for example, reported widespread deactivation of lateral prefrontal cortex (i.e., the DLPFC) and increased activation of the MPFC, a region associated with spontaneous thought processes (e.g., mind wandering; Andrews-Hanna, 2012).

What might account for such discrepant findings? One key methodological difference between Limb and Braun (2008) and others is the sample composition: Limb and Braun (2008) included professional jazz musicians, while most other studies included either classically- 
trained musicians or non-musicians. Studies with classically-trained musicians that report increased activity within executive control regions could simply be a function of increased task demands, since these musicians are presumably less familiar with improvisation. The results of Pinho et al. (2014) suggest that expert improvisers indeed show less activation of lateral prefrontal cortex while improvising compared to novices. However, experts also showed increased functional connectivity between premotor and executive control regions, which suggests that although experts show less activation within lateral prefrontal cortex, they also show greater connectivity between such regions during improvisation. Pinho and colleagues posit that this pattern may reflect a greater automaticity of cognitive processes in experts (cf. Pressing, 1998).

Another possibility is that decreased activation of prefrontal regions reflects greater neural efficiency in experts (cf. Grabner et al., 2006 and Neubauer and Fink, 2009). Because improvisation requires the simultaneous execution of several cognitive processes (Pressing, 1988), the expert improviser, unlike the novice, may be better able to manage these demanding processes, thus reflected in lower levels of metabolic activity in lateral prefrontal cortex. Greater functional connectivity between premotor and executive control regions further supports a neural efficiency hypothesis of improvisational expertise-through practice, musicians may gradually strengthen neural connections, facilitating a more efficient transfer of information between nodes within a larger neural network.

Several other methodological differences may also help to explain disparate patterns of results across the literature. Berkowitz (2010) notes that Limb and Braun (2008) used an experimental design that was less constrained than other studies with classical musicians (e.g., Berkowitz and Ansari, 2008 and Berkowitz and Ansari, 2010). According to Berkowitz (2010), Limb and Braun "allowed their subject-performers to draw on an actual composition for material, with all of its associations-harmonic, melodic, rhythmic, structural, emotions, etc.-in contrast to the much more limited possibilities in our study. Thus, in addition to the active regions shared by our study and theirs, Limb and Braun saw changes in activity in over forty regions” (pp. 142143). Berkowitz (2010) further argues that while the methodological approach of Limb and Braun (2008) may be more similar to real-world improvisation, it may not provide the type of experimental control required to isolate unique neural mechanisms involved in specific improvisational processes (e.g., improvising melodies versus improvising rhythms).

de Manzano and Ullén (2012a) also point to differences in experimental contrasts chosen across studies. Indeed, determining an appropriate baseline condition for contrasting an experimental condition of interest is a fundamental issue in fMRI research (Gusnard and Raichle, 2001). de Manzano and Ullén (2012a) note that several studies that used similar task paradigms, experimental contrasts, and samples reported similar patterns of functional activity, including the DLPFC, pre-SMA, and PMD (Bengtsson et al., 2007,Berkowitz and Ansari, 2008, de Manzano and Ullén, 2012a and Donnay et al., 2014). Moreover, unlikeLimb and Braun (2008), these 
studies did not report activation within default mode regions (i.e., MPFC), nor did they report widespread deactivation of the DLPFC.

Interestingly, Donnay and colleagues did not replicate the results of Limb and Braun (2008). In contrast, they found increased activation of the DLPFC and no significant activation increases in the MPFC. The authors pointed to differences in experimental design between their study and Limb and Braun (2008)-most notably, the addition of a second musician who collaborated with subjects-resulting in greater demands on externally-directed processes associated with executive control (e.g., performance monitoring). On the other hand, musicians in Limb and Braun (2008) did not have such demands on external attention and could thus focus attention on internally-directed processes (e.g., idea generation), possibly reflecting greater involvement of default network regions. Future research could further explore the extent to which the default and executive networks contribute to improvisation by directly contrasting collaborative and individual improvisation.

Although default and executive activity may correspond to greater demands on internally- vs. externally-directed attention, the concurrent activation of both networks' hubs reported in past work suggests that these two networks may not show an antagonistic or "anticorrelated" pattern of activity during improvisation. Instead, such networks may exhibit differential involvement as a function of task demands (cf. Chrysikou et al., 2014). A growing literature points to several cognitive processes associated with large-scale network cooperation, including mind-wandering (Christoff et al., 2009), future planning (Gerlach et al., 2014 and Spreng et al., 2010), and even cognitive control (Cocchi et al., 2013 and Spreng et al., 2014). Default and executive network interaction during improvisation would also be consistent with recent functional connectivity analyses showing cooperation between these networks during other creative thinking tasks, such as divergent thinking (e.g., Beaty et al., 2014a) and artistic drawing (Ellamil et al., 2012. Such tasks are hypothesized to invoke a state of focused internal attention (Andrews-Hanna et al., 2014 and Benedek et al., 2014d), an ability that seems especially relevant to musical improvisation.

\section{Future directions}

In addition to the cognitive processes covered in this review, several non-cognitive, social factors are thought to influence improvisation (e.g., group interaction and audience feedback; Sawyer, 1992 and Sawyer, 2003). McPherson and Limb (2013) raise the question of whether real-world creative performance can even be studied empirically without fundamentally changing the essence of the art. Although constraining musical performance comes at the cost of ecological validity, neuroimaging methods offer powerful tools for isolating specific sub-processes of improvisational cognition while controlling for other related processes. Future researchers should continue to strike a balance between ecological validity and experimental control (cf. Hasson and Honey, 2012). 
Throughout this review, Pressing's (1998) model was used as a framework for interpreting the results of neuroimaging studies on improvisation. Importantly, although the model seems to fit with the literature, the extent to which activation patterns reflect theoretical mechanisms remains unclear. Indeed, one's ability to retrospectively associate brain activity with theory relies on some degree of reverse inference (Poldrack, 2006). Future work should test specific hypotheses related to Pressing's (1988) model and rely less on reverse inferences. To further validate results, researchers could collect behavioral data (e.g., response times) in addition to brain imaging data, which could be used to correlate with activation within a specific region of interest (Poldrack, 2006). Such approaches have already been employed in past work. For example, Bengtsson et al. (2007) assessed the numerical deviation of improvised melodies from predetermined templates and found that as improvised melodies deviated more from the templates, activation within the pre-SMA increased. Future research should use similar approaches to determine the cognitive processes underlying musical improvisation.

Improvisation research should continue to employ both behavioral and neurophysiological techniques to study spontaneous creativity. Several innovative methods have already been developed to study improvisation behaviorally (e.g., Goldman, 2013, Norgaard, 2011, Norgaard, 2014 and Norgaard et al., 2013). Improvisation researchers would also benefit from embracing new and emerging methods in cognitive neuroscience. Like other forms of complex cognition, improvisation involves dynamic communication between regions across the entire cortex. Functional connectivity methods provide useful tools for analyzing the relative influence of nodes within large-scale functional networks (Bullmore and Sporns, 2009 and van den Heuvel and Hulshoff Pol, 2010). Such methods have begun to emerge in the improvisation literature (e.g., Donnay et al., 2014, Pinho et al., 2014 and Wan et al., 2014). Adopting a network-based approach will allow researchers to move beyond analyzing discrete regions in isolation, and transition toward a more fine-grained understanding of the mechanisms underlying musical improvisation.

\section{Acknowledgments}

I thank Mathias Benedek, Donald Hodges, Michael Kane, Jeni Pathman, and Paul Silvia for their comments on this manuscript.

\section{References}

Abraham, A., 2014. Creative thinking as orchestrated by semantic processing vs. cognitive control brain networks. Front. Hum. Neurosci. 8, 95.

Andrews-Hanna, J.R., 2012. The brain's default network and its adaptive role in internal mentation. Neuroscientist 18, 251-270.

Andrews-Hanna, J.R., Smallwood, J., Spreng, R.N., 2014. The default network and selfgenerated thought: component processes, dynamic control, and clinical relevance. 
Ann. N. Y. Acad. Sci. U. S. A. 1316, 29-52. Badre, D., Poldrack, R.A., Pare-Blagoev, E.J., Insler, R.Z., Wagner, A.D., 2005. Dissociable controlled retrieval and generalized selection mechanisms in ventrolateral prefrontal cortex. Neuron 47, 907-918.

Barr, N., Pennycook, G., Stolz, J.A., Fugelsang, J.A., 2014. Reasoned connections: a dualprocess perspective on creative thought. Think. Reason. 21, 61-75.

Beaty, R. E., Benedek, M., Kaufman, S. B., \& Silvia, P. J., 2014a. Default and executive network coupling supports creative idea production, Submitted for publication.

Beaty, R.E., Benedek, M., Wilkins, R.W., Jauk, E., Fink, A., Silvia, P.J., Neubauer, A.C., 2014b. Creativity and the default mode network: a functional connectivity analysis of the creative brain at rest. Neuropsychologia 64, 92-98.

Beaty, R.E., Silvia, P.J., 2012. Why do ideas get more creative across time? An executive interpretation of the serial order effect in divergent thinking tasks. Psychol. Aesthet. Creat. Arts 6, 309-319.

Beaty, R.E., Silvia, P.J., 2013. Metaphorically speaking: cognitive abilities and the production of figurative language. Mem. Cogn. 41, 255-267.

Beaty,R.E., Silvia, P.J.,Nusbaum, E.C.,Jauk, E.,Benedek,M., 2014c. The roles of associative and executive processes in creative cognition. Mem. Cogn. 42, 1186-1197.

Beaty, R.E., Smeekens, B.A., Silvia, P.J., Hodges, D.A., Kane, M.J., 2013. A first look at the role of domain-general cognitive and creative abilities in jazz improvisation. Psychomusicol. Music Mind Brain 23, 262-268.

Benedek, M., Beaty, R.E., Jauk, E., Koschutnig, K., Fink, A., Silvia, P.J., Neubauer, A.C., 2014. Creating metaphors: the neural basis of figurative language production. NeuroImage 90, 99-106.

Benedek, M., Borovnjak, B., Neubauer, A.C., Kruse-Weber, S., 2014a. Creativity and personality in classical, jazz and folk musicians. Personal. Individ. Differ. 63, 117-121.

Benedek, M., Jauk, E., Fink, A., Koschutnig, K., Reishofer, G., Ebner, F., Neubauer, A.C., 2014b. To create or to recall? Neural mechanisms underlying the generation of creative new ideas. NeuroImage 88, 125-133.

Benedek, M., Jauk, E., Sommer, M., Arendasy, M., Neubauer, A.C., 2014c. Intelligence, creativity, and cognitive control: the common and differential involvement of executive functions in intelligence and creativity. Intelligence 46, 73-83.

Benedek, M., Schickel, R.J., Jauk, E., Fink, A., Neubauer, A.C., 2014d. Alpha power increases in right parietal cortex reflects focused internal attention. Neuropsychologia 56, 393-400. 
Bengtsson, S.L., Csikszentmihalyi, M., Ullén, F., 2007. Cortical regions involved in the generation of musical structures during improvisation in pianists. J. Cogn. Neurosci. 19, 830.

Bengtsson, S., Ehrsson, H.H., Forssberg, H., Ullén, F., 2004. Dissociating brain regions controlling the temporal and ordinal structure of learned movement sequences. Eur. J. Neurosci. 19, 2591-2602.

Bengtsson, S.L., Ehrrson, H.H., Hashimoto, T., Kito, T., Ullén, F., Naito, E., Sadato, N., 2009. Listening to rhythms activates motor and premotor cortex. Cortex 45, 62-71.

Berkowitz, A.L., 2010. The Improvising Mind: Cognition and Creativity in the Musical Moment. Oxford University Press, Oxford, UK.

Berkowitz, A.L., Ansari, D., 2008. Generation of novel motor sequences: the neural correlates of musical improvisation. NeuroImage 41, 535-543.

Berkowitz, A.L., Ansari, D., 2010. Expertise-related deactivation of the right temporoparietal junction during musical improvisation. NeuroImage 49, 712-719.

Beudel,M.,de Jong,B.M.,2009. Overlap and segregation in predorsal premotor cortex activations related to free selection of self-referenced and target-based finger movements. Cereb. Cortex 19, 2361-2371.

Bischoff-Grethe, A., Goeder, K.M., Willingham, D.T., Grafton, S.T., 2004. Neural substrates of response-based sequence learning using fMRI. J. Cogn. Neurosci. 16, 127-138.

Botvinick, M.M., Cohen, J.D., Carter, C.S., 2004. Conflict monitoring and anterior cingulate cortex: an update. Trends Cogn. Sci. 8, 539-546.

Brown, S., Martinex, M.J., Parsons, L.M., 2006. Music and language side by side in the brain: a PET study of the generation of melodies and sentences. Eur. J. Neurosci. 23, 2791-2803.

Buckner, R.L., Carroll, D.C., 2007. Self-projection and the brain. Trends Cogn. Sci. 11, 49-57.

Bullmore, E., Sporns, O., 2009. Complex brain networks: graph theoretical analysis of structural and functional systems. Nat. Rev. Neurosci. 10, 186-198.

Christoff, K., Gordon, A.M., Smallwood, J., 2009. Experience sampling during fMRI reveals default network and executive system contributions to mind wandering. Proc. Natl. Acad. Sci. U. S. A. $106,8719-8724$.

Chrysikou, E.G.,Weber, M.J., Thompson-Schill, S.L., 2014.A matched filter hypothesis for cognitive control. Neuropsychologia 62, 341-355.

Cocchi, L., Zalesky, A., Fornito, A., Mattingley, J.B., 2013. Dynamic cooperation and competition between brain systems during cognitive control. Trends Cogn. Sci. 17, 494-501. 
Corbetta, M., Patel, G., Schulman, G.L., 2008. The reorienting system of the human brain: from environment to theory of mind. Neuron 58, 306-324.

Corbetta, M., Shulman, G.L., 2002. Control of goal-directed and stimulus-driven attention in the brain. Nat. Rev. Neurosci. 3, 201-215.

de Manzano, Ö., Ullén, F., 2012a. Activation and connectivity patterns of the presupplementary and dorsal premotor areas during free improvisation of melodies and rhythms. NeuroImage 63, 272-280.

de Manzano, Ö., Ullén, F., 2012b. Goal-independent mechanisms for free response generation: creative and pseudo-random performance share neural substrates. NeuroImage 59, 772-780.

Dietrich, A., Kanso, R., 2010. A review of EEG, ERP, and neuroimaging studies of creativity and insight. Psychol. Bull. 136, 822-848.

Donnay, G.F.,Rankin, S.K., Lopez-Gonzalez,M.,Jiradejvong, P., Limb, C.J., 2014. Neural substrates of interactive musical improvisation: an fMRI study of 'trading fours' in jazz. PLOS ONE 3, e88665.

Dreisbach, G., Haider, H., 2009. How task representations guide attention: further evidence for the shielding function oftask sets. J. Exp. Psychol. Learn. Mem. Cogn. 35, 477-486. Ellamil, M., Dobson, C., Beeman, M., Christoff, K., 2012. Evaluative and generative modes of thought during the creative process. NeuroImage 59, 1783-1794.

Ericsson, K.A., 2013. Training history, deliberate practice and elite sports performance: an analysis in response to Tucker and Collins review-what makes champions? Br. J. Sports Med. 47, 533-535.

Ericsson, K.A., Krampe, R., Tesch-Römer, C., 1993. The role of deliberate practice in the acquisition of expert performance. Psychol. Rev. 100, 363-406.

Fink, A., Benedek, M., 2014. EEG alpha power and creative ideation. Neurosci. Biobehav. Rev. 44, 111-123.

Fink, A., Grabner, R.H., Benedek, M., Reishofer, G., Hauswirth, V., Fally, M., Neubauer, A.C., 2009. The creative brain: investigation of brain activity during creative problem solving by means of EEG and fMRI. Hum. Brain Map. 30, 734-748.

Forstmann, B.U., Brass, M., Koch, O., von Cramon, D.Y., 2006. Voluntary selection of task sets revealed by functional magnetic resonance imaging. J. Cogn. Neurosci. 18, 388-398. 
Gerlach, K.D., Spreng, R.N., Madore, K.P., Schacter, D.L., 2014. Future planning: default network activity coupes with frontoparietal control network and reward-processing regions during process and outcome simulations. Soc. Cogn. Affect. Neurosci. 12, 1942-1951.

Goldman, A., 2013. Towards a cognitive-scientific research program for improvisation: theory and an experiment. Psychomusicol. Music Mind Brain 23, 210-221.

Gonen-Yaacovi, G., de Souza, L.C., Levy, R., Urbanski, M., Josse, G., Volle, E., 2013. Rostral and caudal prefrontal contribution to creativity: a meta-analysis of functional imaging data. Front. Hum. Neurosci. 7, 465.

Grabner, R.H., Neubauer, A.C., Stern, E., 2006. Superior performance and neural effi- ciency: the impact of intelligence and expertise. Brain Res. Bull. 69, 422-439.

Gusnard, D.A., Raichle, M.E., 2001. Searching for a baseline: functional imaging and the resting human brain. Nat. Rev. Neurosci. 2, 685-694.

Hambrick, D.Z., Oswald, F.L., Altmann, E.M., Meinz, E.J., Gobet, F., Campitelli, G., 2014. Deliberate practice: is that all it takes to become an expert? Intelligence 45, 34-45.

Hambrick, D.Z., Meinz, E.J., 2011. Limits on the predictive power of domain-specific experience and knowledge in skilled performance. Curr. Direct. Psychol. Sci. 20, 275-279.

Hasson, U., Honey, C.J., 2012. Future trends in neuroimaging: neural processes as expressed within real-life contexts. NeuroImage 62, 1272-1278.

Hirshorn, E.A., Thompson-Schill, S.L., 2006. Role of the let inferior frontal gyrus in covert word retrieval: neural correlates of switching during verbal fluency. Neuropsychologia 44, 2457-2557.

Howard-Jones, P.A., Blakemore, S.J., Samuel, E.A., Summers, I.R., Claxton, G., 2005. Semantic divergence and creative story generation: an fMRI investigation. Cogn. Brain Res. 25, 240-250.

Jauk, E., Benedek,M., Neubauer,A.C., 2014. The road to creative achievement: a latent variable model of ability and personality predictors. Eur. J. Pers. 28, 95-105.

Jung, R.E., Mead, B.S., Carrasco, J., Flores, R.A., 2013. The structure of creative cognition in the human brain. Front. Hum. Neurosci. 7, 330. Karabanov, A., Blom, O., Forsman, L., Ullén, F., 2009. The dorsal auditory pathway is involved in performance of both auditory and visual rhythms. NeuroImage 44, 480-488.

Kowatari,Y., Hee Lee, S.,Yamamura, H., Nagamori,Y., Levy, P.,Yamane, S.,Yamamoto, M., 2009. Neural networks involved in artistic creativity. Hum. Brain Map. 30, 1678-1690. 
Lee, C.S., Therriault, D.J., 2013. The cognitive underpinnings of creative thought: A latent variable analysis exploring the roles of intelligence and working memory in three creative thinking processes. Intelligence 41, 306-320.

Lewis, C., Lovatt, P.J., 2013. Breaking away from set patterns of thinking: improvisation and divergent thinking. Think. Skills Creat. 9, 46-58.

Limb, C.L., Braun, A.R., 2008. Neural substrates of spontaneous musical performance. An fMRI study of jazz improvisation. PLoS ONE 3, e1679.

Liu, S., Chow, H.M., Xu, Y., Erkkinen, M.G., Swelt, K.E., Eagle, M.W., Braun, A.R., 2012. Neural correlates of lyrical improvisation: an fMRI study of freestyle rap. Nat. Sci. Rep. 2, 834.

McMillan, R.L., Kaufman, S.B., Singer, J.L., 2013. Ode to positive constructive daydreaming. Front. Psychol. 4, 626. McPherson, M., Limb, C.J., 2013. Difficulties in the neuroscience of creativity: Jazz improvisation and the scientific method. Ann. N. Y. Acad. Sci. 1303, 80-83.

Mednick, S.A., 1962. The associative basis of the creative process. Psychol. Rev. 69, 220-232.

Meinz, E.J., Hambrick, D.Z., 2010. Deliberate practice is necessary but not sufficient to explain individual differences in piano sight-reading skill. Psychol. Sci. 21, 914-919.

Mok, L.W., 2014. The interplay between spontaneous and controlled processing in creative cognition. Front. Hum. Neurosci. 8, 663.

Neubauer, A.C., Fink, A., 2009. Intelligence and neural efficiency. Neurosci. Biobehav. Rev. 33, 1004-1023. Norgaard, M., 2011. Descriptions of improvisational thinking by artist-level jazz musicians. J. Res. Music Educ. 59, 109-127.

Norgaard, M., 2014. How jazz musicians improvise: the central role of auditory and motor patterns. Music Percept. Interdiscip. J. 31, 271-287.

Norgaard, M., Jonathan, S., Marian, M., 2013. Testing cognitive theories by creating a patternbased probabilistic algorithm for melody and rhythm in jazz improvisation. Psychomusicol. Music Mind Brain 23, 243-254.

Nusbaum, E.C., Silvia, P.J., 2011. Are intelligence and creativity really so different? Fluid intelligence, executive processes, and strategy use in divergent thinking. Intelligence 39, 36-45.

Nusbaum, E.C., Silvia, P.J., Beaty, R.E., 2014. Ready, set, create: what instructing people to be creative reveals about the meaning and mechanisms of divergent thinking. Psychol. Aesthet. Creat. Arts 8, 423-432. 
O’Callaghan, C., Shine, J.M., Lewis, S.J., Andrews-Hanna, J.R., Irish, M., 2015. Shaped by our thoughts: a new task to assess spontaneous cognition and its associated neural correlates in the default network. Brain Cogn. 93, 1-10.

Pinho,A., de Manzano, Ö., Fransson, P., Eriksson, H., Ullén, F., 2014. Connecting to create: expertise in musical improvisation is associated with increased functional connectivity between premotor and prefrontal areas.J. Neurosci. 34, 6156-6163.

Plucker, J.A., 1999. Is the proof in the pudding? Reanalyses of Torrance's (1958 to present) longitudinal data. Creat. Res. J. 12, 103-114.

Poldrack, R.A., 2006. Can cognitive processes be inferred from neuroimaging data? Trends Cogn. Sci. 10, 59-63. Pressing, J., 1988. Improvisation: methods and models. In: Sloboda, J.A. (Ed.), Generative Processes in Music: The Psychology of Performance, Improvisation, and Composition. Clarendon Press, Oxford, pp. 129-178.

Pressing, J., 1998. Psychological constraints on improvisational expertise and communication. In: Nettl, B., Russell, M. (Eds.), In the Course of Performance: Studies in the World of Musical Improvisation. University of Chicago Press, Chicago and London, pp. 47-68.

Sawyer,R.K., 1992.Improvisational creativity: ananalysis ofjazzperformance.Creat. Res. J. 5, 253-263. Sawyer, R.K., 2003. Improvised Dialogues: Emergence and Creativity in Conversation. Ablex Publishing, Westport, CT.

Schacter, D.L., Addis, D.R., Hassabis, D., Martin, V.C., Spreng, R.N., Szpunar, K.K., 2012. The future of memory: remembering, imagining, and the brain. Neuron 76, 677-694.

Schubotz, R.I., von Cramon, D.Y., 2001. Interval and ordinal properties of sequences are associated with distinct premotor areas. Cereb. Cortex 11, 210-222.

Silvia, P.J., Beaty, R.E., Nusbaum, E.C., 2013.Verbal fluency and creativity: general and specific contributions of broad retrieval ability (Gr)factors to divergentthinking. Intelligence 41, 323340 .

Simon, H.A., Chase, W.G., 1973. Skill in chess. Am. Sci. 61, 394-403.

Sowden, P.T., Pringle, A., Gabora, L., 2014. The shifting sands of creative thinking: connections to dual-process theory. Think. Reason. 21, 40-60.

Spreng, R.N., DuPre, E., Selarka, D., Garcia, J., Gojkovic, S., Mildner, J., Turner, G., 2014. Goal-congruent default network activity facilitates cognitive control. J. Nuerosci. 34, 1410814114. 
Spreng, R.N., Stevens, W.D., Chamberlain, J.P., Gilmore, A.W., Schacter, D.L., 2010. Default network activity, coupled with the frontoparietal control network, supports goal-directed cognition. NeuroImage 53, 303-317.

Torrance, E.P., 1988. The nature of creativity as manifest in its testing. In: Sternberg, R.J. (Ed.), The Nature of Creativity: Contemporary Psychological Perspectives. Cambridge University Press, New York, pp. 43-75.

Troyer, A.K., Moscovitch, M., Winocur, G., Alexander, M.P., Stuss, D., 1998. Clustering and switching on verbal fluency: the effects of focal frontal- and temporal-lobe lesions. Neuropsychologia 36, 499-504.

Tucker, R., Collins, M., 2012. What makes champions? A review of the relative contribution of genes and training to sporting success. Br. J. Sports Med. 46, 555- 561.

Unsworth, N., Engle, R.W., 2007. The nature of individual differences in working memory capacity: active maintenance in primary memory and controlled search from secondary memory. Psychol. Rev. 114, 104-132.

van den Heuvel, M.P., Hulshoff Pol, H.E., 2010. Exploring the brain network: a review of resting-state fMRI functional connectivity. Eur. Neuropsychopharmacol. 20, 519-534.

Walton, M.E., Devlin, J.T., Rushworth, M.F., 2004. Interactions between decision making and performance monitoring within prefrontal cortex. Nat. Neurosci. 7, 1259-1265.

Wan, X., Crüts, B., Jensen, H., 2014. The causal inference of cortical neural networks during musical improvisation. PLOS ONE 9, e112776. 